\title{
Translating the 2019 AAD-NPF Guidelines of Care for the Management of Psoriasis in Pediatric Patients
}

\author{
Deeti J. Pithadia, MD; Kelly A. Reynolds, MD; Erica B. Lee, MD; Jashin J. Wu, MD
}

\section{PRACTICE POINTS}

- For children, several environmental factors may prompt psoriasis flares, and it is critical to identify and eliminate these triggers.

- Although the use of biologics may be limited by cost and injection phobias in children, they may be an appropriate option for children with moderate to severe psoriasis when other therapies have failed. A growing body of literature is establishing the safety and effectiveness of biologics in children.

- Clinicians should thoroughly educate parents/ guardians on the course of psoriasis and treatment options as well as pay special attention to treatment goals and psychosocial factors that may guide decision-making regarding therapy.

In November 2019, the American Academy of Dermatology (AAD) and the National Psoriasis Foundation (NPF) released their recommendations regarding management of psoriasis in pediatric patients. The guidelines include information on quantification of disease severity, identification of triggers, assessment of comorbidities, and therapeutics specific to children with psoriasis. This review aims to highlight the most clinically significant considerations for dermatologists when managing pediatric psoriasis.

Cutis. 2020;106:257-260.

In November 2019, the American Academy of Dermatology (AAD) and the National Psoriasis Foundation (NPF) released their first set of recommendations for the management of pediatric psoriasis. ${ }^{1}$ The pediatric guidelines discuss methods of quantifying disease severity in children, triggers and comorbidities, and the efficacy and safety of various therapeutic agents. This review aims to discuss, in a condensed form, special considerations unique to the management of children with psoriasis as presented in the guidelines as well as grade A- and grade B-level treatment recommendations (Table).

\section{Quantifying Psoriasis Severity in Children}

Percentage body surface area (BSA) involvement is the most common mode of grading psoriasis severity, with less than 3\% BSA involvement being considered mild, 3\% to $10 \%$ BSA moderate, and more than 10\% severe disease. In children, the standard method of measuring BSA is the rule of 9's: the head and each arm make up 9\% of the total BSA, each leg and the front and back of the torso respectively each make up $18 \%$, and the genitalia make up $1 \%$. It also is important to consider impact on quality of life, which may be remarkable in spite of limited BSA involvement. The children's dermatology life quality index score may be utilized in combination with affected BSA to determine the burden of psoriasis in context of impact on daily life. This metric is available in both written and cartoon form, and it consists of 10 questions that include variables such as severity of itch, impact on social life, and effects on sleep. Most notably, this tool incorporates pruritus, ${ }^{2}$ which generally is addressed inadequately in pediatric psoriasis.

\section{Triggers and Comorbidities in Pediatric Patients} In children, it is important to identify and eliminate modifiable factors that may prompt psoriasis flares. Infections,

\footnotetext{
Dr. Pithadia is from the Medical College of Georgia, Augusta University. Dr. Reynolds is from the University of Cincinnati College of Medicine, Ohio. Dr. Lee is from the Department of Internal Medicine, Santa Barbara Cottage Hospital, California. Dr. Wu is from the Dermatology Research and Education Foundation, Irvine, California.

Drs. Pithadia, Reynolds, and Lee report no conflict of interest. Dr. Wu is or has been a consultant, investigator, or speaker for AbbVie Inc; Almirall; Amgen; Arcutis Biotherapeutics; Boehringer Ingelheim; Bristol Myers Squibb; Dermavant Sciences Ltd; Dr. Reddy's Laboratories; Eli Lilly and Company; Galderma; Janssen Pharmaceuticals, Inc; LEO Pharma; Novartis; Regeneron Pharmaceuticals; Sanofi Genzyme; Sun Pharmaceutical Industries Ltd; UCB; and Valeant Pharmaceuticals North America LLC.

The eTable is available in the Appendix online at www.mdedge.com/dermatology

Correspondence: Jashin J. Wu, MD (jashinwu@gmail.com).

doi:10.12788/cutis.0115
} 


\section{Key Considerations Unique to the Management of Pediatric Patients With Psoriasis ${ }^{2-11}$}

Pediatric psoriasis severity may be quantified objectively using the rule of 9's to approximate body surface area involvement and subjectively using the children's dermatology life quality index score

Major triggers for cutaneous flares in children include infections, emotional stress, and cigarette smoke exposure

Psoriatic arthritis in children is associated with development of rheumatologic symptoms years prior to onset of appearance of cutaneous plaques

Children with psoriasis should be routinely assessed for obesity and metabolic risk factors - dyslipidemia and hypertension -in accordance with the American Academy of Pediatrics guidelines ${ }^{8}$; if history and physical examination findings show potential risk, screening for cardiovascular risk factors should be pursued

Pediatric patients with psoriasis may experience shaming and bullying, which may bring on psychosocial comorbidities

Off-label use of topical corticosteroids along with topical vitamin D analogues should be utilized in children with localized mild to moderate disease; combination calcipotriene-betamethasone dipropionate ointment may be applied for up to 4 weeks at a time to the body in children aged 12 years and older; combination calcipotriene-betamethasone dipropionate suspension may be applied for up to 8 weeks at a time to the scalp in children aged 12 years and older

Narrowband UVB therapy is the form of phototherapy with most evidence for use in children and may be combined with coal tar preparations

Methotrexate, cyclosporine, and acitretin may be utilized in children with moderate to severe psoriasis, and pretreatment considerations and monitoring mirror those for adults; because acitretin is not immunosuppressive, important live vaccinations may be administered to children taking this therapy; bony changes that may impede appropriate skeletal development are a major concern in young children on high doses of acitretin

Etanercept is approved for patients with psoriasis aged 4 years and older and ustekinumab for patients aged 6 years and older; infliximab and adalimumab may be utilized off label for patients resistant to these treatments; pretherapy considerations and monitoring are similar to those in adults, though use of these therapies in children may be limited by injection phobias

Clinicians should thoroughly educate children and guardians on the disease, paying special attention to psychosocial factors that may guide decision-making by families regarding therapy

particularly group A beta-hemolytic streptococcal infections, are a major trigger in neonates and infants. Other exacerbating factors in children include emotional stress, secondhand cigarette smoke, Kawasaki disease, and withdrawal from systemic corticosteroids.

Psoriatic arthritis (PsA) is a burdensome comorbidity affecting children with psoriasis. The prevalence of joint disease is 15-times greater in children with psoriasis vs those without, ${ }^{3}$ and $80 \%$ of children with PsA develop rheumatologic symptoms, which typically include oligoarticular disease and dactylitis in infants and girls and enthesitis and axial joint involvement in boys and older children, years prior to the onset of cutaneous disease. ${ }^{4}$ Uveitis often occurs in children with psoriasis and PsA but not in those with isolated cutaneous disease.

Compared to unaffected children, pediatric patients with psoriasis have greater prevalence of metabolic and cardiovascular risk factors during childhood, including central obesity, hypertension, hypertriglyceridemia, hypercholesterolemia, insulin resistance, atherosclerosis, arrythmia, and valvular heart disease. Family history of obesity increases the risk for early-onset development of cutaneous lesions, 5,6 and weight reduction may alleviate severity of psoriasis lesions. ${ }^{7}$ In the United States, many of the metabolic associations observed are particularly robust in Black and Hispanic children vs those of other races. Furthermore, the prevalence of inflammatory bowel disease is 3- to 4-times higher in children with psoriasis compared to those without.

As with other cutaneous diseases, it is important to be aware of social and mental health concerns in children with psoriasis. The majority of pediatric patients with psoriasis experience name-calling, shaming, or bullying, and many have concerns from skin shedding and malodor. Independent risk for depression after the onset of psoriasis is high. Affected older children and adolescents are at increased risk for alcohol and drug abuse as well as eating disorders.

Despite these identified comorbidities, there are no unique screening recommendations for arthritis, ophthalmologic disease, metabolic disease, cardiovascular disease, gastrointestinal tract disease, or mental health issues in children with psoriasis. Rather, these patients should be monitored according to the American Academy of Pediatrics or American Diabetes Association guidelines for all pediatric patients. ${ }^{8,9}$ Nonetheless, educating patients and guardians about these potential issues may be warranted.

\section{Topical Therapies}

For children with mild to moderate psoriasis, topical therapies are first line. Despite being off label, topical corticosteroids are the mainstay of therapy for localized psoriatic plaques in children. Topical vitamin D analogues - calcitriol and calcipotriol/calcipotriene-are highly effective and well 
tolerated, and they frequently are used in combination with topical corticosteroids. Topical calcineurin inhibitors, namely tacrolimus, also are used off label but are considered first line for sensitive regions of the skin in children, including the face, genitalia, and body folds. There currently is limited evidence for supporting the use of the topical vitamin A analogue tazarotene in children with psoriasis, though some consider its off-label use effective for pediatric nail psoriasis. It also may be used as an adjunct to topical corticosteroids to minimize irritation.

Although there is no gold standard topical regimen, combination therapy with a high-potency topical steroid and topical vitamin D analogue commonly is used to minimize steroid-induced side effects. For the first 2 weeks of treatment, they each may be applied once daily or mixed together and applied twice daily. For subsequent maintenance, topical calcipotriene may be applied on weekdays and topical steroids only on weekends. Combination calcipotriol-betamethasone dipropionate also is available as cream, ointment, foam, and suspension vehicles for use on the body and scalp in children aged 12 years and older. Tacrolimus ointment $0.1 \%$ may be applied in a thin layer up to twice daily. Concurrent emollient use also is recommended with these therapies.

Health care providers should educate patients and guardians about the potential side effects of topical therapies. They also should provide explicit instructions for amount, site, frequency, and duration of application. Topical corticosteroids commonly result in burning on application and may potentially cause skin thinning and striae with overuse. Topical vitamin D analogues may result in local irritation that may be improved by concurrent emollient use, and they generally should be avoided on sensitive sites. Topical calcineurin inhibitors are associated with burning, stinging, and pruritus, and the US Food and Drug Administration has issued a black-box warning related to risk for lymphoma with their chronic intermittent use. However, it was based on rare reports of lymphoma in transplant patients taking oral calcineurin inhibitors; no clinical trials to date in humans have demonstrated an increased risk for malignancy with topical calcineurin inhibitors. ${ }^{10}$ Tazarotene should be used cautiously in females of childbearing age given its teratogenic potential.

Children younger than 7 years are especially prone to suppression of the hypothalamic-pituitary-adrenal axis from topical corticosteroid therapy and theoretically hypercalcemia and hypervitaminosis D from topical vitamin D analogues, as their high BSA-to-volume ratio increases potential for systemic absorption. Children should avoid occlusive application of topical vitamin D analogues to large areas of the skin. Monitoring of vitamin D metabolites in the serum may be considered if calcipotriene or calcipotriol application to a large BSA is warranted.

\section{Light-Based Therapy}

In children with widespread psoriasis or those refractory to topical therapy, phototherapy may be considered. Narrowband UVB (311- to 313-nm wavelength) therapy is considered a first-line form of phototherapy in pediatric psoriasis. Mineral oil or emollient pretreatment to affected areas may augment the efficacy of UV-based treatments. ${ }^{11}$ Excimer laser and UVA also may be efficacious, though evidence is limited in children. Treatment is recommended to start at 3 days a week, and once improvement is seen, the frequency can be decreased to 2 days a week. Once desired clearance is achieved, maintenance therapy can be continued at even longer intervals. Adjunctive use of tar preparations may potentiate the efficacy of phototherapy, though there is a theoretical increased risk for carcinogenicity with prolonged use of coal tar. Side effects of phototherapy include erythema, blistering hyperpigmentation, and pruritus. Psoralen is contraindicated in children younger than 12 years. All forms of phototherapy are contraindicated in children with generalized erythroderma and cutaneous cancer syndromes. Other important pediatric-specific considerations include anxiety that may be provoked by UV light machines and inconvenience of frequent appointments.

\section{Nonbiologic Systemic Therapies}

Systemic therapies may be considered in children with recalcitrant, widespread, or rapidly progressing psoriasis, particularly if the disease is accompanied by severe emotional and psychological burden. These drugs, which include methotrexate, cyclosporine, and acitretin (see eTable for recommended dosing), are advantageous in that they may be combined with other therapies; however, they have potential for dangerous toxicities.

Methotrexate is the most frequently utilized systemic therapy for psoriasis worldwide in children because of its low cost, once-weekly dosing, and the substantial amount of long-term efficacy and safety data available in the pediatric population. It is slow acting initially but has excellent longterm efficacy for nearly every subtype of psoriasis. The most common side effect of methotrexate is gastrointestinal tract intolerance. Nonetheless, adverse events are rare in children without prior history, with 1 large study $(\mathrm{N}=289)$ reporting no adverse events in more than $90 \%$ of patients aged 9 to 14 years treated with methotrexate. ${ }^{12}$ Current guidelines recommend monitoring for bone marrow suppression and elevated transaminase levels 4 to 6 days after initiating treatment. ${ }^{1}$ The absolute contraindications for methotrexate are pregnancy and liver disease, and caution should be taken in children with metabolic risk factors. Adolescents must be counseled regarding the elevated risk for hepatotoxicity associated with alcohol ingestion. Methotrexate therapy also requires $1 \mathrm{mg}$ folic acid supplementation 6 to 7 days a week, which decreases the risk for developing folic acid deficiency and may decrease gastrointestinal tract intolerance and hepatic side effects that may result from therapy.

Cyclosporine is an effective and well-tolerated option for rapid control of severe psoriasis in children. It is useful for various types of psoriasis but generally is reserved for more severe subtypes, such as generalized pustular psoriasis, erythrodermic psoriasis, and uncontrolled plaque psoriasis. Long-term use of cyclosporine may result in renal toxicity and hypertension, and this therapy is absolutely 
contraindicated in children with kidney disease or hypertension at baseline. It is strongly recommended to evaluate blood pressure every week for the first month of therapy and at every subsequent follow-up visit, which may occur at variable intervals based on the judgement of the provider. Evaluation before and during treatment with cyclosporine also should include a complete blood cell count, complete metabolic panel, and lipid panel.

Systemic retinoids have a unique advantage over methotrexate and cyclosporine in that they are not immunosuppressive and therefore are not contraindicated in children who are very young or immunosuppressed. Children receiving systemic retinoids also can receive routine live vaccinesmeasles-mumps-rubella, varicella zoster, and rotavirus - that are contraindicated with other systemic therapies. Acitretin is particularly effective in pediatric patients with diffuse guttate psoriasis, pustular psoriasis, and palmoplantar psoriasis. Narrowband UVB therapy has been shown to augment the effectiveness of acitretin in children, which may allow for reduced acitretin dosing. Pustular psoriasis may respond as quickly as 3 weeks after initiation, whereas it may take 2 to 3 months before improvement is noticed in plaque psoriasis. Side effects of retinoids include skin dryness, hyperlipidemia, and gastrointestinal tract upset. The most severe long-term concern is skeletal toxicity, including premature epiphyseal closure, hyperostosis, periosteal bone formation, and decreased bone mineral density. ${ }^{1}$ Vitamin A derivatives also are known teratogens and should be avoided in females of childbearing potential. Lipids and transaminases should be monitored routinely, and screening for depression and psychiatric symptoms should be performed frequently. ${ }^{1}$

When utilizing systemic therapies, the objective should be to control the disease, maintain stability, and ultimately taper to the lowest effective dose or transition to a topical therapy, if feasible. Although no particular systemic therapy is recommended as first line for children with psoriasis, it is important to consider comorbidities, contraindications, monitoring frequency, mode of administration (injectable therapies elicit more psychological trauma in children than oral therapies), and expense when determining the best choice.

\section{Biologics}

Biologic agents are associated with very high to total psoriatic plaque clearance rates and require infrequent dosing and monitoring. However, their use may be limited by cost and injection phobias in children as well as limited evidence for their efficacy and safety in pediatric psoriasis. Several studies have established the safety and effectiveness of biologics in children with plaque psoriasis (see eTable for recommended dosing), whereas the evidence supporting their use in treating pustular and erythrodermic variants are limited to case reports and case series. The tumor necrosis factor $\alpha$ (TNF- $\alpha$ ) inhibitor etanercept has been approved for use in children aged 4 years and older, and the IL-12/IL-23 inhibitor ustekinumab is approved in children aged 6 years and older. Other TNF- $\alpha$ inhibitors, namely infliximab and adalimumab, commonly are utilized off label for pediatric psoriasis. The most common side effect of biologic therapies in pediatric patients is injection-site reactions. ${ }^{1}$ Prior to initiating therapy, children must undergo tuberculosis screening either by purified protein derivative testing or IFN- $\gamma$ release assay. Testing should be repeated annually in individuals taking TNF- $\alpha$ inhibitors, though the utility of repeat testing when taking biologics in other classes is not clear. High-risk patients also should be screened for human immunodeficiency virus and hepatitis. Follow-up frequency may range from every 3 months to annually, based on judgement of the provider. In children who develop loss of response to biologics, methotrexate can be added to the regimen to attenuate formation of efficacy-reducing antidrug antibodies.

\section{Final Thoughts}

When managing children with psoriasis, it is important for dermatologists to appropriately educate guardians and children on the disease course, as well as consider the psychological, emotional, social, and financial factors that may direct decision-making regarding optimal therapeutics. Dermatologists should consider collaboration with the child's primary care physician and other specialists to ensure that all needs are met.

These guidelines provide a framework agreed upon by numerous experts in pediatric psoriasis, but they are limited by gaps in the research. There still is much to be learned regarding the pathophysiology of psoriasis; the risk for developing comorbidities during adulthood; and the efficacy and safety of certain therapeutics, particularly biologics, in pediatric patients with psoriasis.

\section{REFERENCES}

1. Menter A, Cordoro KM, Davis DMR, et al. Joint American Academy of Dermatology-National Psoriasis Foundation guidelines of care for the management and treatment of psoriasis in pediatric patients [published online November 5, 2019]. J Am Acad Dermatol. 2020;82:161-201.

2. Lewis-Jones MS, Finlay AY. The Children's Dermatology Life Quality Index (CDLQI): initial validation and practical use. Br J Dermatol. 1995;132:942-949.

3. Augustin M, Radtke MA, Glaeske G, et al. Epidemiology and comorbidity in children with psoriasis and atopic eczema. Dermatology. 2015;231:35-40.

4. Osier E, Wang AS, Tollefson MM, et al. Pediatric psoriasis comorbidity screening guidelines. JAMA Dermatol. 2017;153:698-704.

5. Boccardi D, Menni S, LaVecchia C, et al. Overweight and childhood psoriasis. Br J Dermatol. 2009;161:484-486.

6. Becker L, Tom WL, Eshagh $\mathrm{K}$, et al. Excess adiposity preceding pediatric psoriasis. JAMA Dermatol. 2014;150:573-574.

7. Alotaibi HA. Effects of weight loss on psoriasis: a review of clinical trials. Cureus. 2018;10:E3491.

8. Guidelines summaries-American Academy of Pediatrics. Guideline Central website. https://www.guidelinecentral.com/summaries/organizations lamerican-academy-of-pediatrics/2019. Accessed October 27, 2020.

9. Standards of Medical Care in Diabetes. American Diabetes Association website. https://care.diabetesjournals.org/content/43 /Supplement_1. Published January 1, 2020. Accessed May 8, 2020.

10. Siegfried EC, Jaworski JC, Hebert AA. Topical calcineurin inhibitors and lymphoma risk: evidence update with implications for daily practice. Am J Clin Dermatol. 2013;14:163-178.

11. Jain VK, Bansal A, Aggarwal K, et al. Enhanced response of childhood psoriasis to narrow-band UV-B phototherapy with preirradiation use of mineral oil. Pediatr Dermatol. 2008;25:559-564.

12. Ergun T, Seckin Gencosmanoglu D, Alpsoy E, et al. Efficacy, safety and drug survival of conventional agents in pediatric psoriasis: a multicenter, cohort study. J Dermatol. 2017;44:630-634. 


\section{APPENDIX}

eTABLE. Recommended Dosing Regimens of the Most Commonly Utilized Systemic Therapies for Pediatric Psoriasis

\begin{tabular}{|c|c|c|c|}
\hline Therapeutic & Modes Available & Group & Dosage \\
\hline \multirow[t]{2}{*}{ Methotrexate } & \multirow{2}{*}{$\begin{array}{l}\text { Oral tablets (may be split } \\
\text { and crushed into food } \\
\text { for younger children); } \\
\text { solution (may be injected } \\
\text { or administered orally) }\end{array}$} & Age $<13$ y & $\begin{array}{l}0.2-0.7 \mathrm{mg} / \mathrm{kg} / \mathrm{wk} \text { (maximum of } 25 \mathrm{mg} / \mathrm{wk} \text { ), titrate } \\
1.25-5 \mathrm{mg} / \mathrm{wk} \text { until effective or maximum dosage is attained }\end{array}$ \\
\hline & & Age $\geq 13$ y & 10-25 mg once weekly \\
\hline Acitretin & $\begin{array}{l}\text { Oral capsules (may be } \\
\text { split and crushed into } \\
\text { food for younger children) }\end{array}$ & All children & $0.1-1 \mathrm{mg} / \mathrm{kg} / \mathrm{d}$ \\
\hline Cyclosporine & $\begin{array}{l}\text { Oral microemulsion } \\
\text { capsules; oral solution }\end{array}$ & All children & $\begin{array}{l}2-5 \mathrm{mg} / \mathrm{kg} / \mathrm{d} \text { administered in } 2 \text { divided doses; initiate at } \\
\text { the higher range of daily dose spectrum and reduce to } \\
\text { lowest effective dose once disease is controlled }\end{array}$ \\
\hline \multirow[t]{2}{*}{ Etanercept } & \multirow[t]{2}{*}{ Subcutaneous injection } & Weight $<63 \mathrm{~kg}$ & $0.8 \mathrm{mg} / \mathrm{kg} / \mathrm{wk}$ \\
\hline & & Weight $\geq 63 \mathrm{~kg}$ & $\begin{array}{l}50 \text { mg twice weekly for } 12 \text { consecutive wk, then } \\
\text { maintenance with } 50 \text { mg once weekly }\end{array}$ \\
\hline Adalimumab & Subcutaneous injection & All children & $\begin{array}{l}24 \mathrm{mg} / \mathrm{m}^{2} \text { or } 0.8 \mathrm{mg} / \mathrm{kg} / \mathrm{wk} \text { (maximum } 40 \mathrm{mg} \text { ) for first } \\
2 \mathrm{wk} \text {, then maintenance with } 0.8 \mathrm{mg} / \mathrm{kg} / \mathrm{wk} \text { every } 2 \mathrm{wk}\end{array}$ \\
\hline Infliximab & Intravenous infusion & All children & $5 \mathrm{mg} / \mathrm{kg}$ at wk 0,2 , and 6 , then maintenance every $8 \mathrm{wk}$ \\
\hline \multirow[t]{3}{*}{ Ustekinumab } & \multirow[t]{3}{*}{ Subcutaneous injection } & Weight $<60 \mathrm{~kg}$ & $\begin{array}{l}0.75 \mathrm{mg} / \mathrm{kg} \text { at wk } 0 \text { and } 4 \text {, then maintenance with } \\
0.75 \mathrm{mg} / \mathrm{kg} \text { every } 12 \mathrm{wk}\end{array}$ \\
\hline & & Weight 60-100 kg & $\begin{array}{l}45 \mathrm{mg} \text { at wk } 0 \text { and } 4 \text {, then maintenance with } \\
45 \mathrm{mg} \text { every } 12 \mathrm{wk}\end{array}$ \\
\hline & & Weight $>100 \mathrm{~kg}$ & $\begin{array}{l}90 \mathrm{mg} \text { at wk } 0 \text { and } 4 \text {, then maintenance with } \\
90 \mathrm{mg} \text { every } 12 \mathrm{wk}\end{array}$ \\
\hline
\end{tabular}

